\title{
Sustained Running in Rats Administered Corticosterone Prevents the Development of Depressive Behaviors and Enhances Hippocampal Neurogenesis and Synaptic Plasticity Without Increasing Neurotrophic Factor Levels
}

\author{
Suk-Yu Yau, $* \star_{*}^{1}$ Ang Li, $* \dagger \S$ En-Dong Zhang, ${ }^{*}$ Brian R. Christie,, $\mathbb{I}$ \\ Aimin Xu, $\$ \S * * *$ Tatia M. C. Lee, $\uparrow+\uparrow+\neq$ and Kwok-Fai So $* \dagger \ddagger \S \S$ III \\ *Department of Anatomy, The University of Hong Kong, Pokfulam, Hong Kong \\ $†$ State Key Laboratory of Brain and Cognitive Science, The University of Hong Kong, Pokfulam, Hong Kong \\ $\ddagger$ Research Centre of Heart, Brain, Hormone and Healthy Ageing, Li Ka Shing Faculty of Medicine, \\ The University of Hong Kong, Pokfulam, Hong Kong \\ $\S$ Department of Medicine, The University of Hong Kong, Pokfulam, Hong Kong SAR \\ IIDivision of Medical Sciences, The University of Victoria, Victoria, British Columbia, Canada \\ \#Department of Pharmacology and Pharmacy, The University of Hong Kong, Pokfulam, Hong Kong \\ **State Key Laboratory of Pharmaceutical Biotechnology, The University of Hong Kong, Pokfulam, Hong Kong \\ $\dagger$ Cognitive Affective Neuroscience, The University of Hong Kong, Pokfulam, Hong Kong \\ \$ Laboratory of Neuropsychology, The University of Hong Kong, Pokfulam, Hong Kong \\ $\S \S$ Department of Ophthalmology, The University of Hong Kong, Pokfulam, Hong Kong \\ IIIGHM Institute of CNS Regeneration, Jinan University, Guangzhou, China
}

\begin{abstract}
We have previously shown that voluntary running acts as an anxiolytic and ameliorates deficits in hippocampal neurogenesis and spatial learning. It also reduces depression-like behaviors that are normally observed in rats that were administered either low $(30 \mathrm{mg} / \mathrm{kg})$ or moderate $(40 \mathrm{mg} / \mathrm{kg})$ doses of corticosterone (CORT). However, the protective effects of running were absent in rats treated with a high $(50 \mathrm{mg} / \mathrm{kg})$ dose of CORT. We examined whether allowing animals to exercise for 2 weeks prior and/or concurrently with the administration of $50 \mathrm{mg} / \mathrm{kg}$ CORT treatment could have similar protective effects. We examined hippocampal neurogenesis using immunohistochemical staining of proliferative and survival cells with the thymidine analogs (BrdU, CIdU, and IdU). In addition, we monitored synaptic protein expression and quantified the levels of neurotrophic factors in these animals as well as performing behavioral analyses (forced swim test and sucrose preference test). Our results indicate that the depressive phenotype and reductions in neurogenesis that normally accompany high CORT administration could only be prevented by allowing animals to exercise both prior to and concurrently with the CORT administration period. These animals also showed increases in both synaptophysin and PSD-95 protein levels, but surprisingly, neither brain-derived neurotrophic factor (BDNF) nor insulin-like growth factor 1 (IGF-1) levels were increased in these animals. The results suggest that persistent exercise can strengthen resilience to stress by promoting hippocampal neurogenesis and increasing synaptic protein levels, thereby reducing the deleterious effects of stress.
\end{abstract}

Key words: Hippocampal neurogenesis; Depression-like behavior; Synaptic plasticity; Physical exercise; Brain-derived neurotrophic factor; Insulin-like growth factor

\section{INTRODUCTION}

It has recently been shown that hippocampal neurogenesis plays a significant role in regulating animal stress responses and depressive behaviors (40). Indeed, it has even been postulated that a decrease in neurogenesis may precipitate the onset of depression (21), although a sustained elevation of glucocorticoids with chronic stress can also severely impair hippocampal neurogenesis, synaptic plasticity, and increase depressive behaviors $(28,37,45)$. Repeated injection of the major stress hormone corticosterone (CORT) in rodents provides a reliable animal model for studying stress-triggered depressive disorders (20). The behavioral changes exhibited by these animals are fairly similar to that of clinical patients, and the

Received November 6, 2013; final acceptance January 28, 2014.

${ }^{1}$ Present address: Division of Medical Sciences, The University of Victoria, Victoria, British Columbia, Canada.

Address correspondence to Professor Kwok-Fai So, Department of Anatomy, Li Ka Shing Faculty of Medicine, The University of Hong Kong, 21 Sassoon Road, Pokfulam, Hong Kong SAR, China. Tel: +852-2819-9216; Fax: +852-2817-0857; E-mail: hrmaskf@ hku.hk or Tatia M. C. Lee, Laboratory of Neuropsychology, Room 656, The Jockey Club Tower, The University of Hong Kong, Hong Kong SAR, China. Tel: +852-3917-8394; Fax: +852-2819-0978; E-mail: tmclee@ hku.hk 
severity of induced behavioral depression parallels the magnitude of the dose of CORT administered $(23,49)$. Our prior work has shown that voluntary wheel running for 2 weeks can decrease the incidence of depression-like behavior in the rats treated with 30 or $40 \mathrm{mg} / \mathrm{kg}$, but not $50 \mathrm{mg} / \mathrm{kg}$ of CORT (49). In addition, voluntary exercise rescued hippocampal neurogenesis in these animals and elevated brain-derived neurotrophic factor (BDNF) levels, indicating some possible mechanisms for the therapeutic effects of exercise in this model.

Although the mechanisms underlying exercise-mediated antidepressant effects have yet to be fully elucidated, increasing hippocampal neurogenesis is associated with a decrease in depressive behaviors $(7,42)$. Interestingly, exercise has also been shown to increase levels of neurotrophic factors like BDNF and insulin-like growth factor 1 (IGF-1) that are important for neuronal survival $(5,19,25)$. Wheel running for 1 week prior to forced swim-induced stress inhibits the downregulation of hippocampal BDNF mRNA as well as prolongs swimming time (1). In addition, combining antidepressant treatments with running can have synergistic effects and increase BDNF mRNA levels more than either alone (35). We hypothesized that prior physical conditioning might also protect the brain from subsequent stress by creating a prolonged increase in neurotrophic factor levels and enhanced neurogenesis.

To examine this hypothesis, we examined 1) whether 2 weeks of prior running could reduce the behavioral deficits that are produced by $50 \mathrm{mg} / \mathrm{kg}$ CORT treatment; 2) whether these behavioral changes are associated with altered hippocampal neurogenesis and synaptic plasticity; and 3) whether there are corresponding changes in hippocampal and serum levels of BDNF and IGF-1.

\section{MATERIALS AND METHODS}

\section{Animals and Housing Conditions}

The experimental procedures were approved by the Committee on the Use of Live Animals in Teaching and Research at the University of Hong Kong. Adult male Sprague-Dawley rats $(250 \pm 20 \mathrm{~g})$ were purchased from the laboratory animal unit of the University of Hong Kong and were housed individually in the cage with a locked wheel or unlocked wheel. All animals were kept on a 12-h light-dark cycle with ad libitum access to food and water. In this study, animals ( $n=4-6 /$ group) were divided into four groups: 1) Control rats without running (CON); 2) CORT-treated rats that were allowed to run only during the 2-week CORT administration period (CR); 3) CORT-treated rats with the 2-week prior running only (PR); 4) CORT-treated rats that were allowed to run both prior and concurrently with the CORT administration period $(\mathrm{PR}+\mathrm{CR})$. The timeline of treatments and animal grouping are illustrated in Figure 1. We have previously documented that nonrunning rats receiving CORT (NR) treatment showed a significant decrease in hippocampal cell proliferation along with increased depressive behavior (49), whereas vehicle-treated running rats $(\mathrm{R}+\mathrm{Vel})$ showed significant increase in hippocampal neurogenesis and decrease in depressive behavior (49). For a ready comparison, the corresponding data of NR and $\mathrm{R}+\mathrm{Vel}$ groups was derived from our previous publications $(49,50)$ and were included in figures as specified.

Rats were treated with sesame oil (Sigma-Aldrich, St Louis, MO, USA) or CORT (50 mg/kg, Sigma-Aldrich) for 2 weeks. The doses of 5-iodo-2'-deoxyuridine (IdU, Sigma-Aldrich; $57.7 \mathrm{mg} / \mathrm{kg}$ body weight) and 5-chloro2'-deoxyuridine (CIdU, Sigma-Aldrich; $42.5 \mathrm{mg} / \mathrm{kg}$ body weight) were applied to label different populations of proliferating cells (17). IdU prepared in $0.9 \%$ saline solution (Sigma-Aldrich) containing $0.01 \% \mathrm{NaOH}$ (SigmaAldrich) was injected intraperitoneally during the last 3 days of the prior running stage to study the survival of cells born before CORT injection, while CIdU (prepared in $0.9 \% \mathrm{NaCl}$ solution) was administered during the last 3 days of CORT treatment to study cell proliferation. Bromodeoxyuridine (BrdU, Sigma-Aldrich; $50 \mathrm{mg} / \mathrm{kg}$ body weight) was dissolved in $0.9 \%$ saline and administered intraperitoneally prior to initiation of prior running.

\section{Administration of CORT}

The stock solution of CORT at the dose of $50 \mathrm{mg} / \mathrm{kg}$ and sesame oil were prepared and injected subcutaneously as described by Hellsten et al. (22). Subcutaneous injection of CORT in the neck region has been reported to increase blood levels of CORT over a period of $24 \mathrm{~h}$ (37). CORT was prepared as a stock by suspending CORT in sesame oil, followed by vortex and sonication. Injection was made daily at 4:00 p.m. continuously for 2 weeks. The control rats received daily injections of sesame oil.

\section{Exercise Training}

Runners were individually housed in cages with unlocked wheels (diameter, $31.8 \mathrm{~cm}$; width, $10 \mathrm{~cm}$; Nalgene Nunc International, Rochester, NY, USA), while nonrunners were housed in similar conditions but with locked wheels. The time points for locking and unlocking manipulations are indicated in Figure 1. Wheel revolutions were recorded at 1-h intervals using the VitalViewer software (Mini Mitter Company, Inc., Bend, OR, USA).

\section{Forced Swim Test (FST)}

Depression-like behavior was measured according to the method of Porsolt et al. (33). Briefly, animals were initially allowed to adapt to the testing room for $2 \mathrm{~h}$ in the afternoon (between 2:00 p.m. and 6:00 p.m.). The rats were then placed in a custom-made cylinder (produced by the workshop of Department of Physiology, the University of Hong Kong, Hong Kong, $60 \mathrm{~cm}$ height $\times 25 \mathrm{~cm}$ 


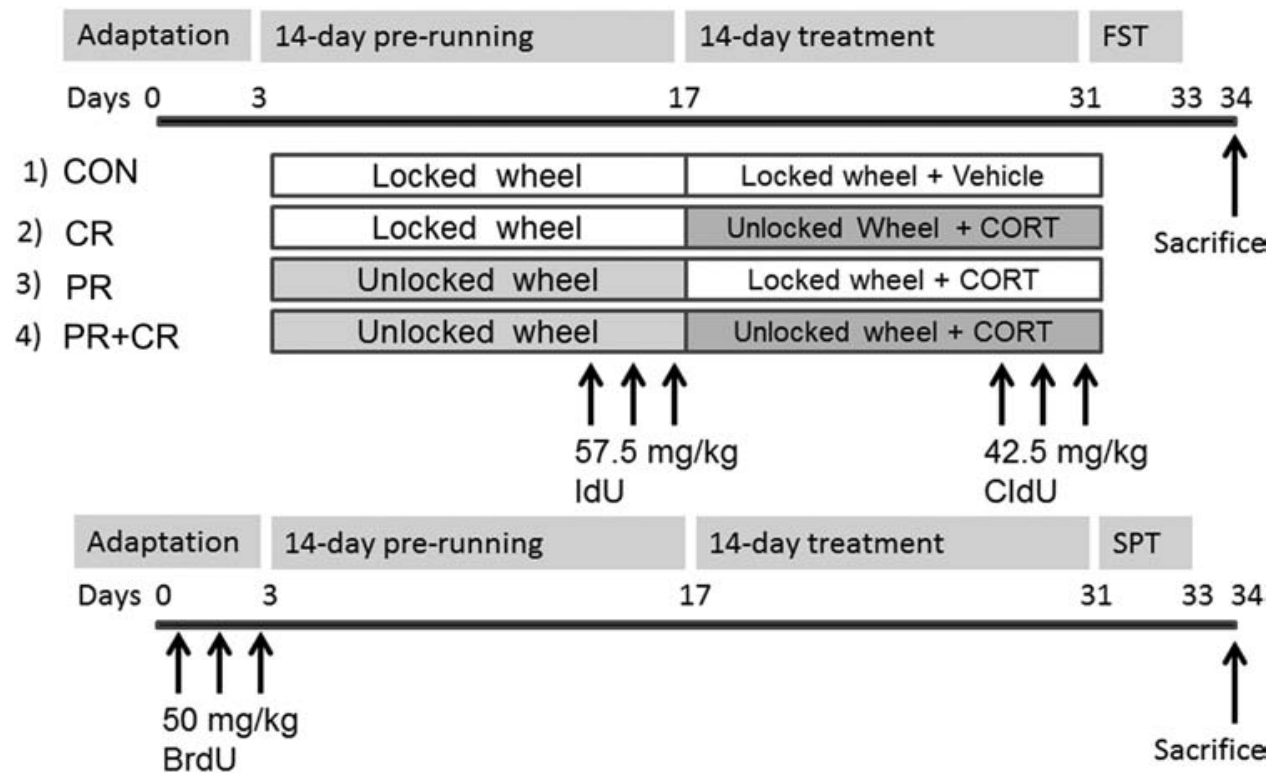

Figure 1. The treatment timeline. The rats were allowed to adapt to a new living environment with a locked wheel in the cage for 2 days. The wheels were then unlocked for the rats with running for 14 days prior to the $50 \mathrm{mg} / \mathrm{kg}$ corticosterone (CORT) treatment. 5-Iodo-2'-deoxyuridine (IdU) was injected to study cell survival, while 5-chloro-2'-deoxyuridine (CIdU) was injected to examine cell proliferation, followed by forced swim test (FST) $24 \mathrm{~h}$ later. Rats injected with bromodeoxyuridine (BrdU) were sacrificed $24 \mathrm{~h}$ after sucrose preference test. The animals were divided into four groups: 1) Control without running (CON); 2) CORT-treated runners that were allowed to exercise during the CORT administration period only (CR); 3) CORT-treated rats with prior running only (PR); 4) CORT-treated rats that were allowed to exercise both prior and concurrently with the CORT administration period ( $P R+C R)$.

diameter) containing tap water for $15 \mathrm{~min}$ on day 1 and 5 min on day 2. Behavioral performance in day 2 was videotaped, and the immobility time was scored with the experimenter blinded to the animal group identity.

\section{Sucrose Preference Test (SPT)}

The rats were simultaneously offered with $1 \%$ sucrose (Sigma-Aldrich) solution and tap water for $24 \mathrm{~h}$. The position of the two bottles was swapped after $12 \mathrm{~h}$. The bottles were weighed before and after the test to calculate the consumption of water and sucrose solution. The sucrose preference was presented as the percentage of sucrose solution to the total amount of liquid consumption. Decrease in sucrose preference indicates the state of anhedonia-like behavior that is frequently seen in depressive patients.

\section{Tissue Preparation}

Animals were euthanized by overdose of pentobarbitone (Alfasan International B.V. Woerden, Holland) and perfused transcardially with $0.9 \% \mathrm{NaCl}$ (Sigma-Aldrich), followed by $4 \%$ paraformaldehyde (International Laboratory, San Francisco, CA, USA). The brain was postfixed at $4{ }^{\circ} \mathrm{C}$ overnight and then left in $30 \%$ sucrose buffer until it sank. Coronal sections of $40-\mu \mathrm{m}$ thickness were cut through the hippocampus from the bregma $-3.30 \mathrm{~mm}$ to $-4.52 \mathrm{~mm}$ (32).

\section{Immunohistochemistry}

Brain sections were bathed in $0.01 \mathrm{M}$ citric acid (USB Corporation, Cleveland, $\mathrm{OH}, \mathrm{USA})(\mathrm{pH}=6.0)$ at $95^{\circ} \mathrm{C}$ for $30 \mathrm{~min}$ in a water bath. After three $0.01 \mathrm{M}$ phosphatebuffered saline (PBS; Sigma-Aldrich) rinses, sections were then incubated in $2 \mathrm{~N} \mathrm{HCl}$ (Sigma-Aldrich) for $30 \mathrm{~min}$ at $37^{\circ} \mathrm{C}$, followed by 15 -min incubation in boric acid buffer (Merck KGaA, Darmstadt, Germany) at room temperature. After thorough rinses in 0.01 M PBS, sections were incubated overnight with mouse anti-BrdU antibody (1:1,000; Roche Molecular Biochemicals, Essex, UK, for detecting IdU and BrdU) or rat anti-BrdU $(1: 1,000$; Abcam, Cambridge, MA, USA, for detecting CIdU) in antibody diluents at room temperature. Sections were washed and incubated with biotinylated goat anti-mouse or anti-rat antibody (1:200; Vector Laboratories, Inc., Burlingame, CA, USA). Finally, sections were incubated with an avidin-biotin complex (Vector Laboratories), and the IdU/CIdU/BrdU immunoreactivity was visualized with liquid diaminobenzidine substrate kit (Invitrogen, Carlsbad, CA, USA). Brain sections were counted in a 1-in-12 series of coronal sections.

For double staining of CIdU and doublecortin (DCX: immature neuronal marker), the sections were concurrently incubated with rat anti-BrdU antibody (1:1,000, Abcam) and rabbit anti-DCX (1:100, Abcam) overnight at room 
temperature. Thereafter, they were probed with Alexa fluor 488-conjugated goat anti-rabbit and 568-conjugated goat anti-rat (1:200, Molecular Probes, Eugene, OR, USA) for $2 \mathrm{~h}$ at room temperature. After rinsing, sections were coverslipped with the mounting medium (Dako, Carpinteria, CA, USA) for later analysis.

\section{Quantification of Cell Proliferation, Neuronal Differentiation, and Cell Survival}

Six coronal brain sections containing IdU or CIdUpositive cells per animal were counted through a $40 \times$ objective using the Stereo Investigator (MicroBrightField, Williston, VT, USA) as previously performed $(49,52)$. The number of positive cells was counted within the granular cell layer (GCL) and two cell diameters below the GCL, ignoring those cells in the uppermost focal plane. The estimated cell count was multiplied by 12 to obtain the estimated total number of IdU-, CIdU-, or BrdU-positive cells. The cell counting was performed in a sample-blind manner.

For immunophenotypic analysis, 30 CIdU-positive cells per animal were randomly selected and their coexpression of CIdU and DCX analyzed in a blinded manner by fluorescence microscopy (Axioplan, Zeiss, Oberkochen, Germany) with 40x magnification. Double staining was defined by CIdU-positive nucleus stained together with DCX-positive surrounding soma in a cell. The proportion of DCX-positive cells over the 30 CIdU-positive cells was used as the indicator for neuronal differentiation as previously performed (50).

\section{Protein Extraction and Western Blot Analysis}

Total proteins were extracted from freshly isolated hippocampi after euthanizing rats. Tissues were lysed with radioimmunoprecipitation assay (RIPA) buffer (Cell Signaling Technology, Danvers, MA, USA) supplemented with the protease and phosphatase inhibitor cocktails and phenylmethanesulfonyl fluoride (Sigma-Aldrich). Samples were homogenized and subsequently sonicated (Branson Sonicator \#2510, Branson Ultrasonics, Danbury, CT, USA) for $20 \mathrm{~s}$ with a $50 \%$ pulse and cleared by centrifugation $(10,000 \times g)$ at $4^{\circ} \mathrm{C}$ for $30 \mathrm{~min}$. Supernatant protein was collected and stored at $-80^{\circ} \mathrm{C}$ until use. Supernatant protein concentrations were determined by the DC Protein assay (Bio-Rad Laboratories, Hercules, CA, USA). Homogenate containing $20 \mu \mathrm{g}$ of protein per lane was separated by sodium dodecyl sulfate-polyacrylamide gel electrophoresis (SDS-PAGE) (Bio-Rad Laboratories) and transferred to polyvinyldifluoride (PVDF) membranes (Bio-Rad Laboratories). Nonspecific binding was blocked with $5 \%$ bovine serum albumin (Sigma-Aldrich) for $1 \mathrm{~h}$. Blots were then probed overnight at $4^{\circ} \mathrm{C}$ with primary antibodies, followed by 1 -h incubation with secondary antibodies conjugated to horseradish peroxidase
(HRP) and then developed by chemiluminescence detection (Luminate Forte; Millipore, Ballerica, MA, USA). The antibodies used for detection were mouse anti- $\beta$ actin (1:7,000; Dako, Glostrup, Denmark), rabbit antisynaptophysin $(1: 3,000)$, and rabbit anti-postsynaptic density 95 (PSD-95; 1:3,000; Cell Signaling Technology); and goat anti-mouse and goat anti-rabbit IgG-HRP (1:5,000, Dako).

\section{Measurement of Serum and Hippocampal $B D N F$ and $I G F-l$}

Animals were anesthetized using the mixture of ketamine and xylazine (2:1 ratio), and blood was collected from the tail vein within 3 min using a 25-gauge needle. Blood samples were placed at room temperature for $30 \mathrm{~min}$, followed by centrifugation (Eppendorf centrifuge 5417 R, Hauppauge, NY, USA) at $1000 \times g$ for $20 \mathrm{~min}$ at $4{ }^{\circ} \mathrm{C}$. Serum aliquots were then stored at $-80^{\circ} \mathrm{C}$ until use. The BDNF and IGF-1 levels were determined using commercially available ELISA kits (BDNF: Chemikine BDNF Sandwich ELISA Kit, Millipore; Mouse/Rat IGF-1 Quantikine ELISA Kit, R \& D System, Minneapolis, MN, USA). Sample neurotrophin levels were calculated from the standard curve transforming the absorbance into neurotrophin concentrations.

\section{BDNF Enzyme-Linked Immunosorbent Assay}

In brief, BDNF standards, $100-\mu$ serum samples in 200-fold dilution and 50- $\mu$ g hippocampal protein sample brought to $100 \mu \mathrm{l}$ using sample diluent were added to a 96-well plate and incubated overnight with shaking at $4^{\circ} \mathrm{C}$. After three washes of the plate with wash buffer, the diluted biotinylated mouse anti-BDNF monoclonal antibody was added to the plate and then incubated at room temperature for $3 \mathrm{~h}$. After washing, the diluted streptavidin-HRP conjugate solution was added and incubated for $1 \mathrm{~h}$ at room temperature. After 15-min incubation with TMB/E substrate, the reaction was stopped by adding stop solution. The absorbance was then measured at $450 \mathrm{~nm}$.

\section{IGF-1 Enzyme-Linked Immunosorbent Assay}

The IGF-1 standard, serum samples in 1,000-fold dilution and $50-\mu \mathrm{g}$ protein that were brought to the same total volume of standard or serum samples by assay diluent were added to a 96-well plate and incubated with $150-\mu \mathrm{l}$ assay diluent for $2 \mathrm{~h}$ at $4^{\circ} \mathrm{C}$. After washes with wash buffer, the plate was incubated with cold IGF-1 conjugate for $1 \mathrm{~h}$ at $4^{\circ} \mathrm{C}$, followed by washings and 30-min incubation with substrate solution at room temperature. The reaction was stopped by adding stop solution, and absorbance was measured at $450 \mathrm{~nm}$ and corrected by subtracting reading at $570 \mathrm{~nm}$. 


\section{Statistical Analysis}

ANOVA analysis with Fisher's post hoc test was applied for all data sets. Values are presented as mean \pm SEM.

\section{RESULTS}

Sustained Running Decreased Depression-Like Phenotype in Rats Treated With $50 \mathrm{mg} / \mathrm{kg}$ CORT

We have previously shown that $50 \mathrm{mg} / \mathrm{kg}$ CORT treatment in nonrunners (NR) significantly increased immobility time in the forced swim test $(49,52)$. Here we observed significant increases in immobility time in the $\mathrm{CR}$ and $\mathrm{PR}$ groups when compared to the $\mathrm{CON}$ group $[F(3,18)=7.366, p<0.005]$ (Fig. 2A), indicating both concurrent running alone and prior running alone could not alleviate CORT-induced depressive behavior. In contrast, continuous running effectively ameliorated such a depressive behavior, as indicated by no significant difference in immobility time between the CON and PR +CR group. Interestingly, rats with prior running showed significantly less immobility time when compared to rats with concurrent running $(p<0.05)$, suggesting prior running alone somehow could reduce depression level to a greater extent when compared to concurrent running alone, though none of them were effective enough to alleviate CORT-induced depressive behavior.

Anhedonia-like behavior, a core symptom of depressive patients, was measured by the sucrose preference test. Similarly, continuous running reversed CORT-suppressed sucrose consumption ( $p>0.05$ compared to the control, and $p<0.05$ compared to the CR group) (Fig. 2B). Prior running only or concurrent running only failed to attenuate CORT-suppressed sucrose consumption $(p<0.05$ compared to the control). The behavioral data suggest that continuous running, but not prior running or concurrent running, could effectively alleviate the depression-like phenotype in rats treated with high dose of CORT.

\section{Continuous Running Ameliorated Decrease in Hippocampal Neurogenesis and Increased Cell Survival in CORT-Treated Rats}

We next examined whether continuous running exerts its therapeutic effect via restoring hippocampal neurogenesis in the CORT-treated rats. ANOVA analysis on CIdU-labeled cells revealed that continuous running restored the decrease in cell proliferation to the control level, whereas prior running or concurrent running alone was unable to reverse the decrease $[F(3,16)=7.254$, $p<0.005$ ] (Fig. 3A). Decreases in cell proliferation were equivalent in the CR, PR, and NR groups (Fig. 3B).

We studied the survival of two different cell populations that were born in different periods. Cells labeled with IdU were used to estimate the survival of cells labeled during the last 3 days of running. The number of IdU-positive cells in rats with prior running or concurrent running was comparable to that of the control group (Fig. 3D). In contrast, the continuous running that the $\mathrm{PR}+\mathrm{CR}$ group engaged in showed significantly increased cell survival ( $p<0.05 \mathrm{CR}$ vs. $\mathrm{PR}+\mathrm{CR}$ group) (Fig. 3D). BrdU labeling was used to examine the survival of cells born before the initiation of prior running. There was no significant difference in number of BrdU-labeled cells $[F(3,19)=0.865, p=0.479]$ (Fig. 3E), suggesting that the survival of this cell population was not affected by the treatments. Interestingly, we observed a significant negative correlation between the number of IdU-positive cells and
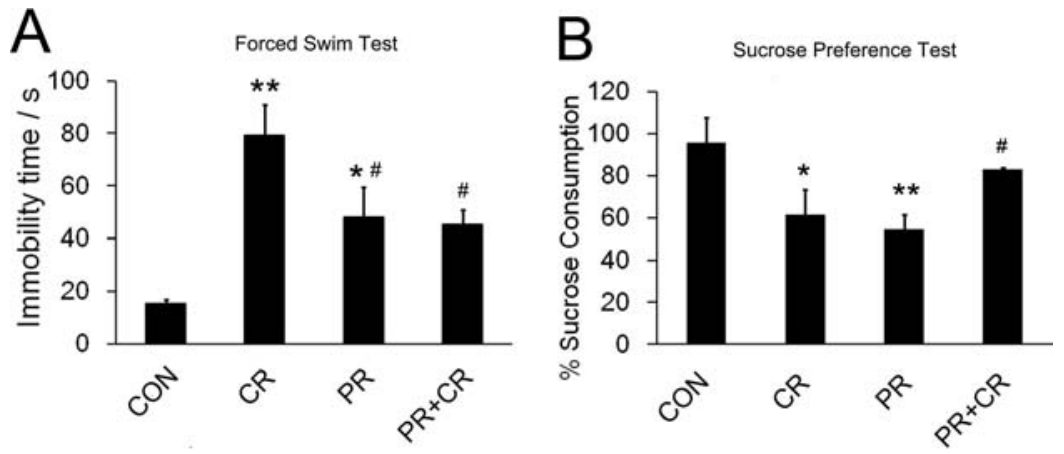

Figure 2. Continuous running decreased depression-like phenotypes. (A) Immobility time was increased in the CR and PR groups compared to the CON group, while there is no significant difference between the CON and PR + CR groups. The PR and PR + CR groups showed that significantly less time was spent in immobility compared to the CR group. **p<0.005, *p<0.05 compared to the control group, $\# p<0.05$ compared to the CR group. (B) Sucrose consumption was significantly decreased in CR and PR groups, whereas the PR +CR group showed equivalent sucrose consumption to the CON group. The PR + CR group showed significant higher sucrose consumption compared to the $\mathrm{CR}$ group. The data indicated that prior running together with concurrent running is required to ameliorate anhedonia-like behavior in rats treated with $50 \mathrm{mg} / \mathrm{kg}$ CORT. $* p<0.05$, ** $p<0.005$ compared to the CON group, \#p<0.05 compared to the CR group. 

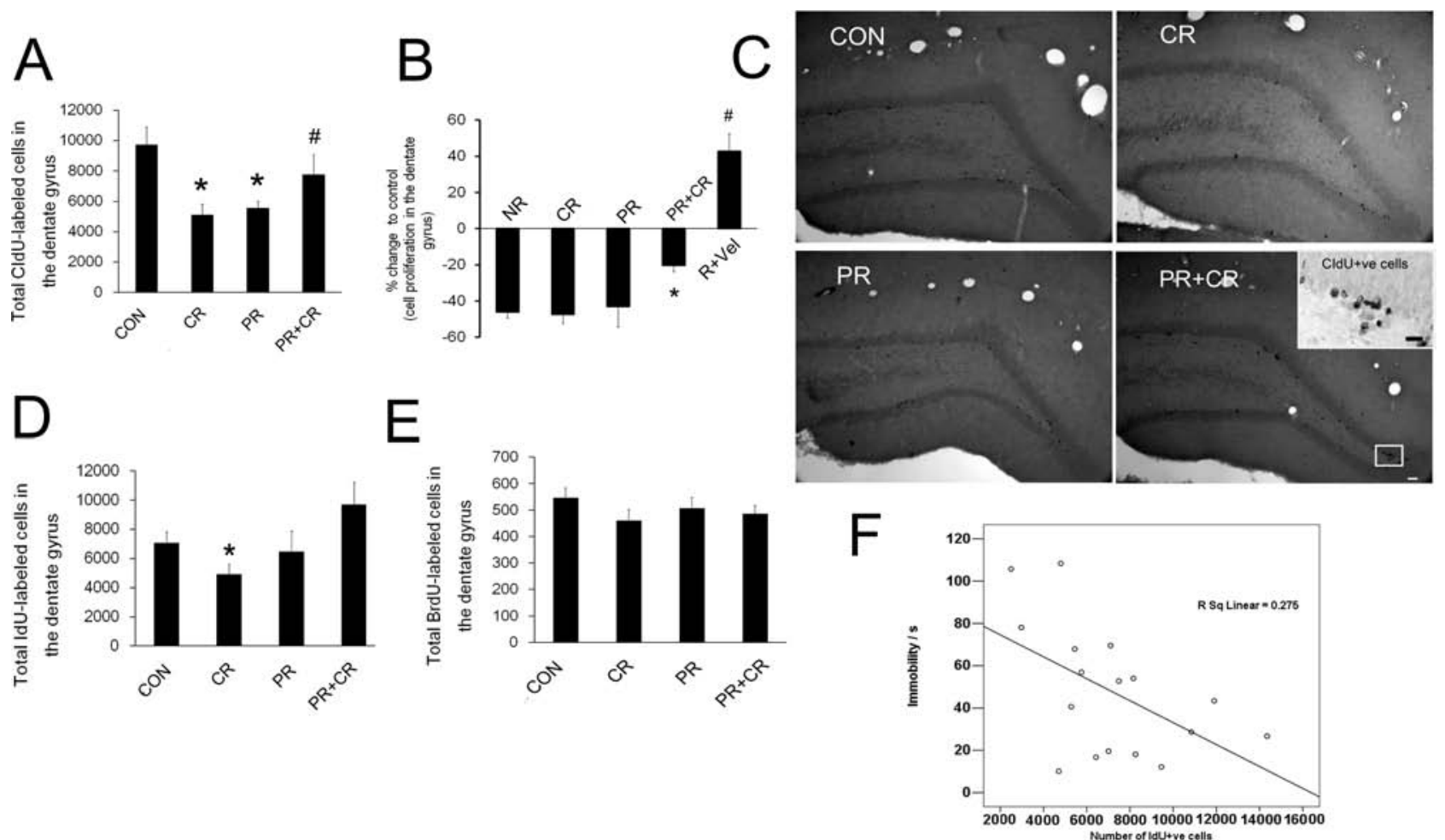

Figure 3. Continuous running ameliorated the decrease in hippocampal neurogenesis in the CORT-treated runners. (A) There was a significant decrease in CIdU-positive cells in the CR and PR groups when compared to the CON group. Conversely, the PR + CR group showed a comparable level of CIdU-positive cells to the control and a significant increase in CIdU positive cells compared to the CR group, indicating continuous running rescued hippocampal cell proliferation in CORT-treated runners. $* p<0.05$ compared to the CON group; $\# p<0.05$ compared to the CR group. (B) Decreases in the number of proliferating cells were equivalent in nonrunning (NR), PR and CR groups, indicating an inability of prior running alone or concurrent alone in rescuing CORT-induced suppression in cell proliferation. Continuous running rescued this decrease as indicated by a significant difference between PR $+\mathrm{CR}$ and $\mathrm{NR}$ or CR or PR groups. ${ }^{*} p<0.05, \# p<0.05$ compared to the other groups. $\mathrm{R}+$ Vel: running and vehicle treated. The data of NR and $\mathrm{R}+\mathrm{Vel}$ groups were derived from our previous publications $(49,50)$. (C) Representative images of CIdU-positive cells in the dentate gyrus. Scale bar: low magnification $50 \mu \mathrm{m}$; high magnification: $25 \mu \mathrm{m}$. (D) The CR group showed a lower trend in the number of surviving cells to the control group. Prior running significantly increased IdU-labeled cells in the PR +CR group compared to the CR group, indicating continuous running increased cell survival. ${ }^{*} p<0.05$ compared to the $\mathrm{PR}+\mathrm{CR}$ group. (E) There was no difference in BrdU-positive cells among the treatment groups. (F) The number of IdU-labeled cells was negatively correlated with the time spent in immobility. The rats with a higher number of IdU-labeled cells showed a lower degree of depression-like behavior in terms of less immobility time. The data were analyzed using Pearson correlation test $\left(R^{2}=0.275, p=0.031\right)$.

time spent in immobility $\left(R^{2}=0.275, p=0.031\right)($ Fig. 3F), suggesting possible involvement of this population of cells in the depressive behavior phenotype in the CORTtreated rats.

Previous data revealed that $50 \mathrm{mg} / \mathrm{kg}$ CORT treatment significantly decreased neuronal differentiation in nonrunners (52). There was a significant increase in the number of cells colabeled with CIdU and the immature neuronal marker DCX in the $\mathrm{CR}$ and $\mathrm{PR}+\mathrm{CR}$ groups $[F(3,22)=4.584, p<0.05]$ (Fig. 4$)$. We subsequently estimated the total number of newborn neurons by multiplying the number of proliferating cells (CIdU-positive cells) to the percentage of newborn cells differentiated into neuronal lineage (the ratio of CIdU-positive cells expressing DCX) (15). The results demonstrated that continuous running prevented the CORT-induced decrease in neurogenesis that was observed in the NR group in our previous study (52). Both the PR and the CR groups showed significant decreases in neurogenesis $[F(3,16)=5.276, p<0.05]$ (Table 1), indicating that prior running together with concurrent running was required to ameliorate the effects of CORT.

\section{Continuous Running and Prior Running Increased Synaptic Plasticity in CORT-Treated Rats}

Running and stress are known to have opposite effects on hippocampal synaptic plasticity $(31,43)$. To examine this issue, we assayed synaptic proteins that are important for synaptic plasticity: synaptophysin and PSD-95 in the hippocampus. Western blot analysis revealed significant changes in protein levels of synaptophysin (Fig. 5B) 


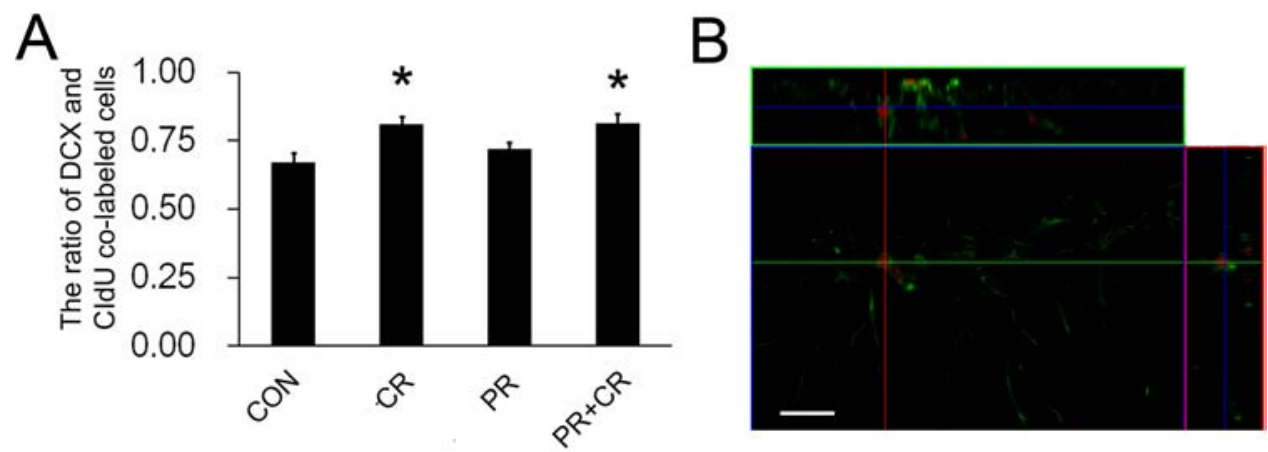

Figure 4. Running during 14-day CORT treatment increased neuronal differentiation. (A) Concurrent running during the administration of CORT treatment increased neuronal differentiation in CR and PR + CR groups when compared to the CON group. Prior running did not affect neuronal differentiation. (B) Representative image of colabeling of CIdU (in red) with doublecortin (in green). * $p<0.05$ compared to the control group. Scale bar: $50 \mu \mathrm{m}$.

$[F(3,22)=30.594, p<0.0005]$ and PSD-95 $[F(3,22=$ $17.476, p<0.0005]$ in the hippocampus.

Continuous running in the $\mathrm{PR}+\mathrm{CR}$ group robustly increased both protein levels in comparison to the CON, $\mathrm{CR}$, and PR groups $(p<0.05)$. Levels for both synaptophysin and PSD-95 in the CR group were significantly decreased ( $p<0.05$ compared to the CON group), indicating that exercise during the CORT administration did not have protective effects for synaptic protein expression. Interestingly, prior running maintained protein levels of synaptophysin and significantly increased levels of PSD-95 when compared to the control group $(p<0.05)$.

\section{Hippocampal and Serum Levels of BDNF and IGF-1 Were not Increased in All CORT-Treated Rats}

The neurotrophins, such as IGF-1 and BDNF, are believed to play an important role in running-induced neurogenesis (11). We examined whether changes of hippocampal and serum IGF-1 and BDNF levels correspond to changes of hippocampal neurogenesis. Hippocampal and serum levels of BDNF [Fig. 6A, hippocampus: $F(3,20)=$ 10.062, $p<0.005$; Fig. 6B, serum: $F(3,20)=5.303, p<0.05]$ and IGF-1 [Fig. 6C, hippocampus: $F(3,20=10.062$, $p<0.005$; Fig. 6D, serum: $F(3,23)=8.927, p<0.005]$ were decreased in both the PR and CR groups. Surprisingly, although the $\mathrm{PR}+\mathrm{CR}$ rats showed elevated hippocampal neurogenesis, they also exhibited a significant decrease in both hippocampal and serum BDNF and IGF-1 levels $(p<0.05)$.

\section{DISCUSSION}

Regular exercise has been well documented to exert preventative or curative effects on depression $(13,41)$; however, the underlying mechanism(s) are not fully clear at present. Our results showed that continuous running in the $\mathrm{PR}+\mathrm{CR}$ group provided protection from the negative effects of CORT administration. Increase in depressive phenotype and reductions in neurogenesis that are induced by high CORT administration could only be prevented in rats with continuous running. These animals also showed increases in both synaptophysin and PSD-95 protein levels, but surprisingly, BDNF and IGF-1 levels were not increased in these animals. These results suggest that sustained physical exercise can protect against stress via rescuing hippocampal neurogenesis and increase synaptic protein contents.

Table 1. Total Number of Newborn Neurons and Surviving Cells

\begin{tabular}{lcccc}
\hline & $\begin{array}{c}\text { CIdU+ Cell } \\
\text { (Proliferating Cells) }\end{array}$ & $\begin{array}{c}\text { DCX/CIdU Ratio } \\
\text { (Neuronal Differentiation) }\end{array}$ & $\begin{array}{c}\text { Number of } \\
\text { Newborn Neurons }\end{array}$ & $\begin{array}{c}\text { IdU+ Cell } \\
\text { (Survival Cells) }\end{array}$ \\
\hline CON & $9,744 \pm 1,002$ & 0.67 & $6,550 \pm 777$ & $7,080 \pm 730$ \\
CR & $5,124 \pm 605^{*}$ & $0.81 \dagger$ & $4,156 \pm 491^{*}$ & $4,929 \pm 678 \ddagger$ \\
PR & $5,558 \pm 357^{*}$ & 0.72 & $3,276 \pm 761^{*}$ & $6,470 \pm 1,400$ \\
PR+CR & $7,770 \pm 1118$ & $0.81 \dagger$ & $6,324 \pm 910$ & $9,705 \pm 1,520$ \\
\hline
\end{tabular}

The number of newborn neurons was obtained by multiplying the total number of CIdU-labeled cells with the ratio of CIdU-labeled cells that expressed the neuronal marker DCX. Combining prior running (PR) and concurrent running (CR) restored the decrease in newborn neurons in the $50 \mathrm{mg} / \mathrm{kg}$ corticosterone (CORT)-treated rats. However, prior running alone was unable to restore CORT suppression of newborn neurons. ${ }^{*} p<0.05$ compared to the control and PR $+\mathrm{CR}$ groups, respectively. $\dagger p<0.05$ compared to CON. $\ddagger p<0.05$ compared to the PR+CR group. DCX: doublecortin; CIdU: 5-chloro-2'-deoxyuridine; IdU: 5-iodo-2'-deoxyuridine. 

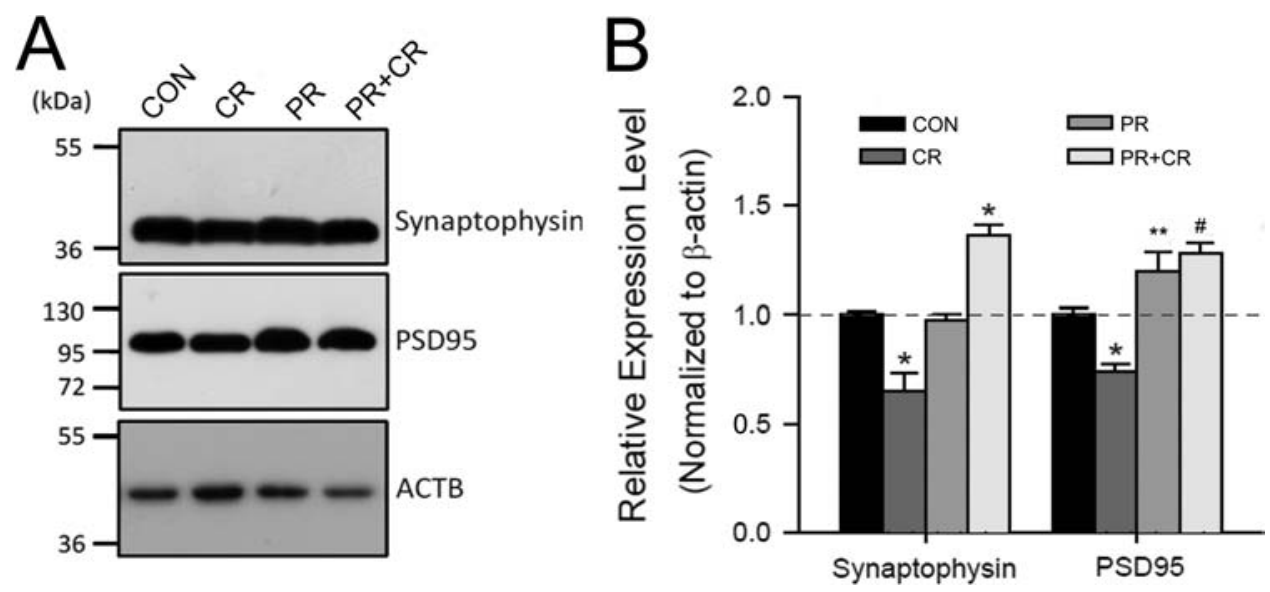

Figure 5. Continuous running increased synaptic protein expressions in CORT-treated rats. (A) Representative bands for Western blot analysis. (B) Significant increases in levels of both synaptophysin and postsynaptic density 95 (PSD 95) were found in the PR + CR group compared to the CON group. Conversely, concurrent running (CR) could not rescue the decrease in synaptophysin and PSD 95 protein expression. Prior running alone maintained synaptophysin protein at the control levels and increased PSD 95 levels. * $p<0.005$ compared to the other three groups respectively; $* * p<0.05 ; \# p<0.005$ compared to the CON group. ACTB: $\beta$ actin.
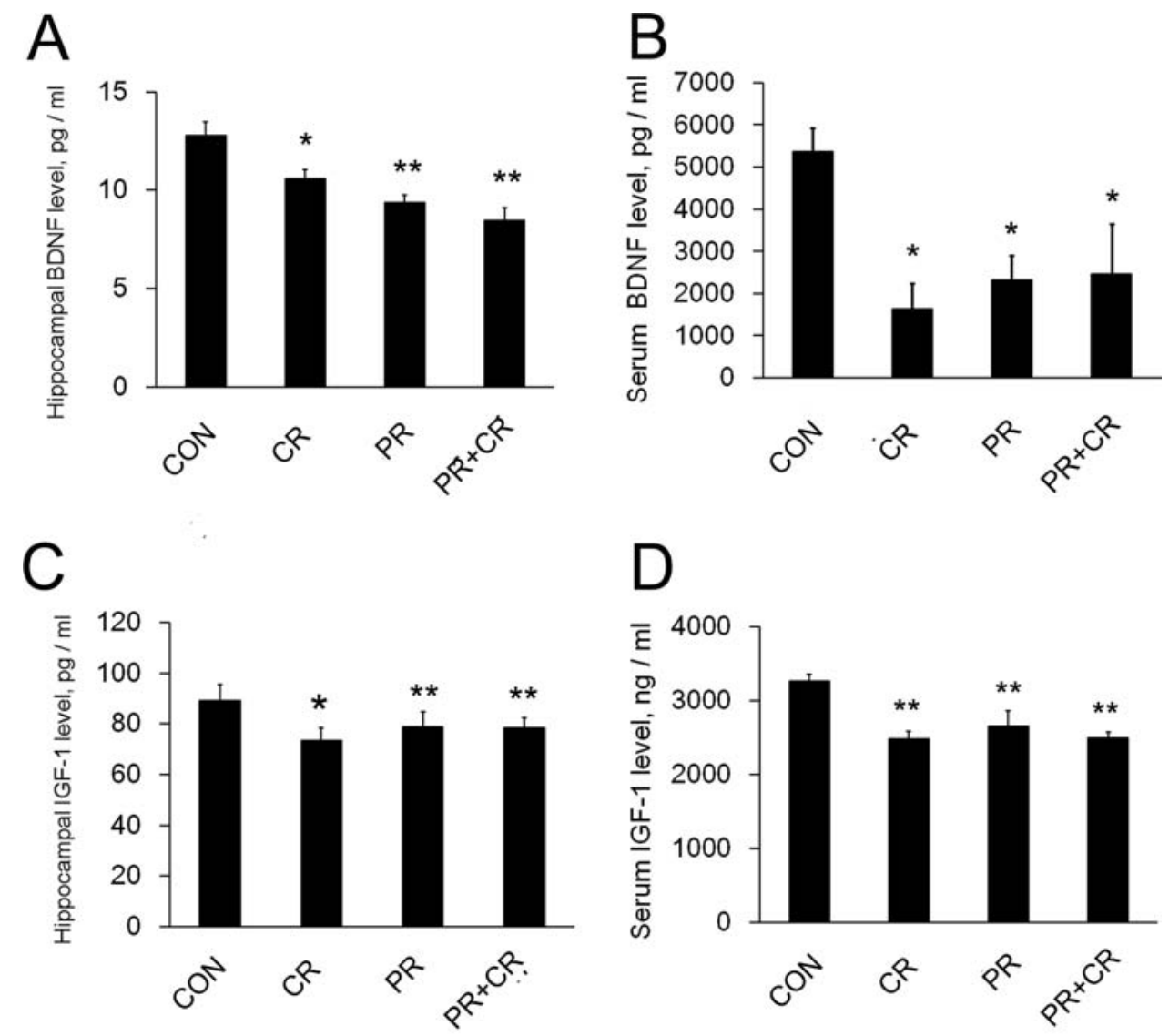

Figure 6. Decreased hippocampal and serum levels of BDNF and IGF in CORT-treated rats. (A) Hippocampal and (B) serum brainderived neurotrophic factor (BDNF) levels were significantly decreased following CORT treatment when compared to the control. (C) Hippocampal and (D) serum insulin-like growth factor-1 (IGF-1) levels were significantly decreased following CORT treatment. $* p<0.05, * * p<0.005$ compared to the CON group. 
Continuous Running Reverses Depression-Like Behavior in Association With Enhanced Cell Proliferation, Neurogenesis, and Cell Survival

Physical exercise is known to increase cell proliferation, neuronal differentiation, and neuronal survival (44), while CORT showed opposite effects (48). With the approach of labeling proliferating cells at different time points, we measured these three indices concurrently. Our previous studies have shown that running enhances hippocampal cell proliferation in rats treated with $20 \mathrm{mg} / \mathrm{kg}$ CORT (51) and counteracts decrease in neurogenesis and depressive behavior induced by 30 and $40 \mathrm{mg} / \mathrm{kg}$ CORT treatment (49). A recent study indicated that running prior to induction of bacterial meningitis increased animal survival and hippocampal neurogenesis (46). We found that only continuous running counteracted high doses of CORT-induced decrease in hippocampal neurogenesis, while prior running or concurrent running alone could not rescue such decrease. The decrease in number of proliferating cells in CORT-treated rats with prior running or concurrent running alone was equivalent to that in CORT-treated rats without running. Reduction of newborn neurons in our rats is likely due to the decrease in proliferating cells, rather than differentiation or survival, since colabeling of CIdU with DCX could be increased following concurrent running, and cell survival rate indicated by the number of IdU- and BrdUpositive cells in rats with prior or concurrent running were comparable to the control group.

Fabel and colleagues observed running-induced increase in adult neurogenesis after 35 days of cessation of running, suggesting that running could prime a survival-promoting effect on newborn cells after cessation of running (15). Conversely, a high dose of CORT suppressed not only cell proliferation but also survival of newborn cells (47). Continuous running considerably increased IdU-positive numbers when compared to the concurrent running group, suggesting prior running together with concurrent running will be required for promoting neuronal survival. Hippocampal neurogenesis has been proposed to mediate the therapeutic effects of antidepressants (such as fluoxetine) (36) and physical exercise $(7,38,42)$ in animal models of depression. The present study further supports this functional role of hippocampal neurogenesis as CORTtreated animals with decreased hippocampal neurogenesis showed depression-like phenotypes, whereas CORT-treated rats with continuous running showed improvement in depressive-like phenotype along with enhanced hippocampal neurogenesis.

\section{Synaptic Plasticity Is Required for Running-Elicited Antidepressant Effect in Stressed Rats}

Our CORT-treated rats with prior running showed significantly less time spent on immobility when compared to that with concurrent running, indicating that prior running potentially reduces the severity of depression-like behavior. A significant correlation between the number of IdU-positive cells and time spent on immobility (Fig. 3F) suggested that these surviving cells may be partially involved in depressive phenotype in the rats, as newborn neurons could be functionally integrated into preexisting circuitry before they become fully mature (29). The beneficial effect of prior running may also partially be fulfilled by enhanced synaptic plasticity since prior running maintained synaptophysin protein levels and enhanced protein PSD-95 levels. PSD-95 is abundantly located at the postsynaptic site to facilitate dendritic spine formation and stabilization $(34,39)$. Overexpression of PSD-95 could increase spine formation and enhance synaptic connection with the presynaptic terminals (30). Decrease in both synaptophysin and PSD-95 by CORT treatment accordingly may indicate the reduced synaptic contact and connection within the hippocampal circuit under stress. Concurrent running could not reverse a CORT-induced decrease in hippocampal neurogenesis and synaptic proteins, which may consequently lead to disrupted hippocampal function and subsequent behavioral deficits in CORT-treated rats. Prior running also failed to reduce depression-like phenotype in CORT-treated rats, which is likely due to decreased hippocampal neurogenesis, despite there being an increase in levels of synaptic protein PSD-95. Conversely, continuous running showed its antidepressant effect together with restored hippocampal neurogenesis and increased synaptic proteins. These observations echo our previous finding and substantiate our hypothesis that both dendritic plasticity and neurogenesis are required to maintain hippocampal function under stress condition (49).

\section{Continuous Running Improves Hippocampal Plasticity in the Presence of Decreased Neurotrophins}

BDNF and IGF-1 have been recognized as the important mediators for running-induced hippocampal neurogenesis, synaptic plasticity, and cognitive improvement (12). Benefits of preexposure to running or antidepressant have been shown to prevent decrease in hippocampal BDNF levels and increase in immobility time $(1,35)$. BDNF has been reported to elevate after running for 28 days in young mice (2), which remained at significantly high levels even 1-2 weeks after the cessation of exercise (6). We expect to observe an increase in both BDNF and IGF-1 levels in the rats with continuous running. Interestingly, we found that continuous running ameliorated the CORT effect in concurrence with decreases in both hippocampal and serum levels of BDNF and IGF-1. Our previous study has suggested that mild changes of neurotrophins in the brain may not be reflected by changes in blood levels (50). The present study demonstrated a corresponding change in central and peripheral levels of neurotrophins, suggesting that significant changes of 


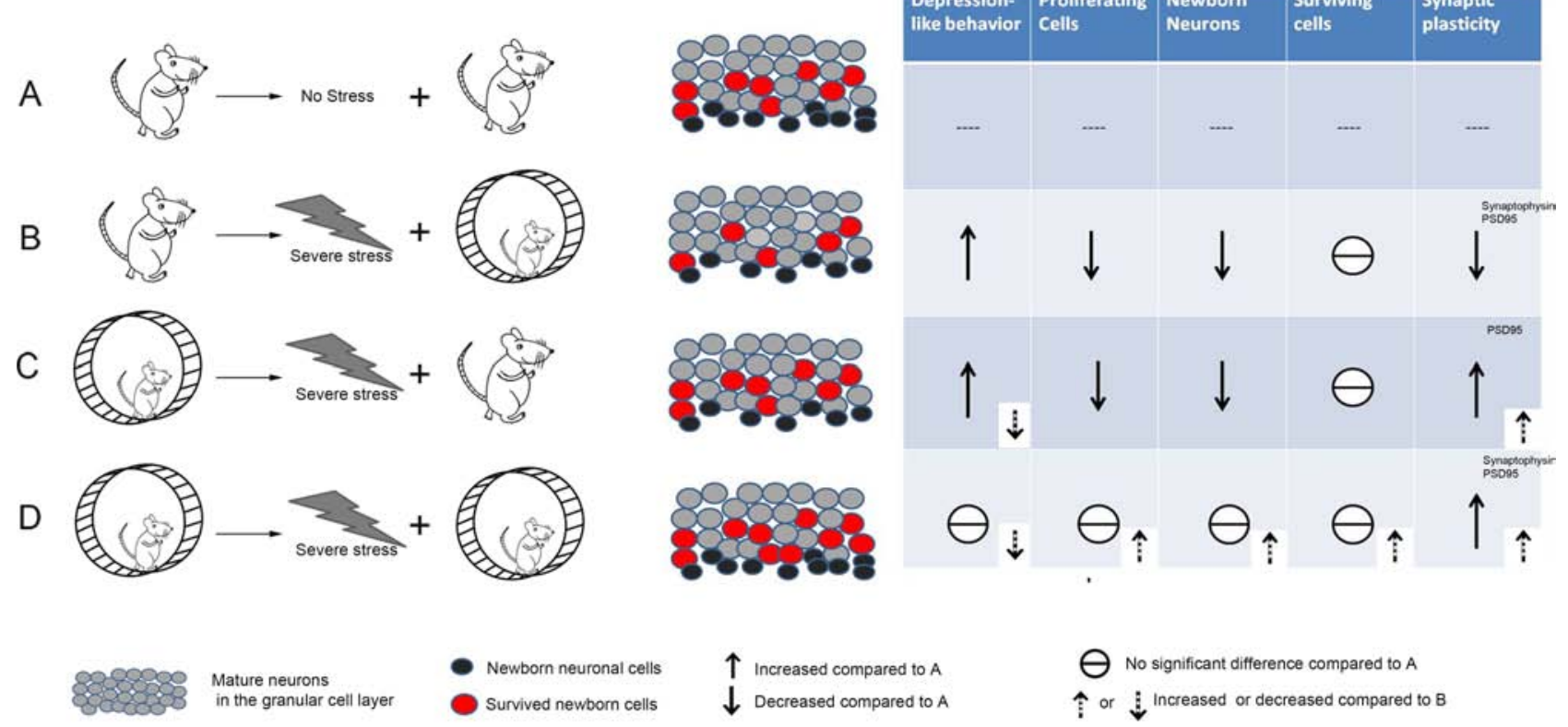

Figure 7. Continuous running prevents depression via enhancing hippocampal neurogenesis and levels of synaptic proteins. (A) The vehicle-treated nonrunner rats served as basal level for comparison. (B) Under severe conditions with $50 \mathrm{mg} / \mathrm{kg}$ CORT treatment, concurrent running is unable to reduce depression-like phenotype and restore both hippocampal neurogenesis and synaptic plasticity. (C) Prior running cannot ameliorate CORT-induced increase in depression-like phenotype, which may be linked to decrease in hippocampal neurogenesis. (D) Continuous running prevents depressive behavior; this beneficial effect may be mediated by rescued hippocampal neurogenesis and enhanced synaptic plasticity.

neurotrophins in the brain could correspond to changes in the blood. However, the present study suggests that these changes may not necessarily correlate with changes in hippocampal plasticity.

To the best of our knowledge, this is the first report showing that increased neurogenesis and enhanced synaptic plasticity could coexist with decrease in hippocampal and serum levels of neurotrophic factors. BDNF levels are increased at week 4 , then return to baseline at week 8 in multiple sclerosis subjects (24). Most clinical studies have shown a transient increase in peripheral BDNF levels following an acute aerobic exercise; however, there is no clinical study showing a long-lasting response of peripheral BDNF to physical training (10). Long-term physical exercise is known to improve cognitive function in normal subjects or subjects with dementia (3). However, decrease in serum BDNF (9) and IGF-1 levels (14) have been reported in normal subjects with chronic physical training. An inverse relationship between cardiorespiratory fitness and serum BDNF levels has also been reported $(16,41)$. The similar finding has been observed in our recent study showing a negative correlation between neurotrophic factors and cognitive functions studied in adolescent athletes (26). Our previous work has shown a decreasing trend in peripheral IGF-1 levels following 4 weeks of running; however, these rats showed a significant increase in hippocampal neurogenesis together with enhanced hippocampal-dependent spatial learning and memory following 2 weeks or 4 weeks of running $(49,50)$. Taken together, our data may suggest that a constantly elevated level of neurotrophin is not always required for enhanced hippocampal neurogenesis and/or synaptic plasticity following prolonged physical exercise.

We hypothesized that over a long time when animals have chronic exercise, other types of events take place, which would result in positive influence on the brain. These changes may result in a more long-lasting beneficial effect that is independent of changes in neurotrophin levels at later time points of physical exercise. Treadmill exercise could enhance brain levels of tropomyosinrelated kinase B (TrkB, BDNF receptor) and synaptic protein in association with enhanced memory in mice (18). The observed beneficial effects of continuous running on our CORT-treated rats may be due to upregulated receptor levels of neurotrophin factors, which may in turn enhance receptor-triggered downstream signaling even in presence of low concentrations of neurotrophins.

\section{Long-Term Physical Exercise as Preventative Measure for Stress-Related Mood Disorders}

Physical exercise is an effective therapeutic intervention for depressed patients $(4,8,13)$. Marlatt et al. (27) 
have shown that the neurogenic response to exercise in mice is much stronger than that to antidepressant treatment with fluoxetine or duloxetine. Regular physical exercise potentially serves as the most effective nonpharmacological tool to enhance brain health. Maintaining an active lifestyle will be a preventative measure to reduce risk of stress-related disorders or enhance the resilience of our body to cope with stress responses in daily life. Taken together, our results suggest that lifelong physical exercise might be a useful practice for preventing stressrelated mood disorders via maintaining hippocampal neurogenesis and synaptic plasticity (Fig. 7).

ACKNOWLEDGMENTS: This study was supported by funding from Jessie Ho Professorship in Neuroscience (The University of Hong Kong Foundation for Educational Development and Research Limited), funding from the State Key Laboratory of Brain and Cognitive Science, the University of Hong Kong, and the fundamental research funds for the central universities (09ykpy25, 09ykpy31), and the postdoctoral fellowship to S. Y. Yau from Research Centre of Heart, Brain, Hormone and Healthy Ageing, Li Ka Shing Faculty of Medicine, the University of Hong Kong. The authors declare no conflicts of interest.

\section{REFERENCES}

1. Adlard, P. A.; Cotman, C. W. Voluntary exercise protects against stress-induced decreases in brain-derived neurotrophic factor protein expression. Neuroscience 124(4): 985-992; 2004.

2. Adlard, P. A.; Perreau, V. M.; Engesser-Cesar, C.; Cotman, C. W. The timecourse of induction of brain-derived neurotrophic factor mRNA and protein in the rat hippocampus following voluntary exercise. Neurosci. Lett. 363(1):43-48; 2004.

3. Ahlskog, J. E.; Geda, Y. E.; Graff-Radford, N. R.; Petersen, R. C. Physical exercise as a preventive or disease-modifying treatment of dementia and brain aging. Mayo. Clinic Proceedings 86(9):876-884; 2011.

4. Babyak, M.; Blumenthal, J. A.; Herman, S.; Khatri, P.; Doraiswamy, M.; Moore, K.; Craighead, W. E.; Baldewicz, T. T.; Krishnan, K. R. Exercise treatment for major depression: Maintenance of therapeutic benefit at 10 months. Psychosom. Med. 62(5):633-638; 2000.

5. Barnabe-Heider, F.; Miller, F. D. Endogenously produced neurotrophins regulate survival and differentiation of cortical progenitors via distinct signaling pathways. J. Neurosci. 23(12):5149-5160; 2003.

6. Berchtold, N. C.; Castello, N.; Cotman, C. W. Exercise and time-dependent benefits to learning and memory. Neuroscience 167(3):588-597; 2010.

7. Bjornebekk, A.; Mathe, A. A.; Brene, S. The antidepressant effect of running is associated with increased hippocampal cell proliferation. Int. J. Neuropsychopharmacology 8(3): 357-368; 2005.

8. Blumenthal, J. A.; Babyak, M. A.; Moore, K. A.; Craighead, W. E.; Herman, S.; Khatri, P.; Waugh, R.; Napolitano, M. A.; Forman, L. M.; Appelbaum, M.; Doraiswamy, P. M.; Krishnan, K. R. Effects of exercise training on older patients with major depression. Archives of Internal Medicine 159(19):2349-2356; 1999.

9. Colcombe, S. J.; Erickson, K. I.; Scalf, P. E.; Kim, J. S.; Prakash, R.; McAuley, E.; Elavsky, S.; Marquez, D. X.;
Hu, L.; Kramer, A. F. Aerobic exercise training increases brain volume in aging humans. J. Gerontol. A. Biol. Sci. Med. Sci. 61(11):1166-1170; 2006.

10. Colcombe, S. J.; Kramer, A. F.; Erickson, K. I.; Scalf, P.; McAuley, E.; Cohen, N. J.; Webb, A.; Jerome, G. J.; Marquez, D. X.; Elavsky, S. Cardiovascular fitness, cortical plasticity, and aging. Proc. Natl. Acad. Sci. U S A 101(9): 3316-3321; 2004.

11. Cotman, C. W.; Berchtold, N. C. Exercise: A behavioral intervention to enhance brain health and plasticity. Trends Neurosci. 25(6):295-301; 2002.

12. Cotman, C. W.; Berchtold, N. C.; Christie, L. A. Exercise builds brain health: Key roles of growth factor cascades and inflammation. Trends Neurosci. 30(9):464-472; 2007.

13. Daley, A. Exercise and depression: A review of reviews. J. Clin. Psychol. Med. Settings 15(2):140-147; 2008.

14. Erickson, K. I.; Raji, C. A.; Lopez, O. L.; Becker, J. T.; Rosano, C.; Newman, A. B.; Gach, H. M.; Thompson, P. M.; Ho, A. J.; Kuller, L. H. Physical activity predicts gray matter volume in late adulthood: The Cardiovascular Health Study. Neurology 75(16):1415-1422; 2010.

15. Fabel, K.; Wolf, S. A.; Ehninger, D.; Babu, H.; Leal-Galicia, P.; Kempermann, G. Additive effects of physical exercise and environmental enrichment on adult hippocampal neurogenesis in mice. Front. Neurosci. 3:50; 2009.

16. Frazer, C. J.; Christensen, H.; Griffiths, K. M. Effectiveness of treatments for depression in older people. Medical Journal of Australia 182(12):627-632; 2005.

17. Gobeske, K. T.; Das, S.; Bonaguidi, M. A.; Weiss, C.; Radulovic, J.; Disterhoft, J. F.; Kessler, J. A. BMP signaling mediates effects of exercise on hippocampal neurogenesis and cognition in mice. PLoS One 4(10):e7506; 2009.

18. Gomes da Silva, S.; Unsain, N.; Masco, D. H.; ToscanoSilva, M.; de Amorim, H. A.; Silva Araujo, B. H.; Simoes, P. S.; Naffah-Mazzacoratti Mda, G.; Mortara, R. A.; Scorza, F. A.; Cavalheiro, E. A.; Arida, R. M. Early exercise promotes positive hippocampal plasticity and improves spatial memory in the adult life of rats. Hippocampus 22(2):347$358 ; 2012$.

19. Gould, E.; Reeves, A. J.; Fallah, M.; Tanapat, P.; Gross, C. G.; Fuchs, E. Hippocampal neurogenesis in adult Old World primates. Proc. Natl. Acad. Sci. U S A 96(9):52635267; 1999.

20. Gregus, A.; Wintink, A. J.; Davis, A. C.; Kalynchuk, L. E. Effect of repeated corticosterone injections and restraint stress on anxiety and depression-like behavior in male rats. Behav. Brain Res. 156(1):105-114; 2005.

21. Hanson, N. D.; Owens, M. J.; Nemeroff, C. B. Depression, antidepressants, and neurogenesis: A critical reappraisal. Neuropsychopharmacology 36(13):2589-2602; 2011.

22. Hellsten, J.; Wennstrom, M.; Mohapel, P.; Ekdahl, C. T.; Bengzon, J.; Tingstrom, A. Electroconvulsive seizures increase hippocampal neurogenesis after chronic corticosterone treatment. Eur. J. Neurosci. 16(2):283-290; 2002.

23. Johnson, S. A.; Fournier, N. M.; Kalynchuk, L. E. Effect of different doses of corticosterone on depression-like behavior and HPA axis responses to a novel stressor. Behav. Brain Res. 168(2):280-288; 2006.

24. Kramer, A. F.; Erickson, K. I. Capitalizing on cortical plasticity: Influence of physical activity on cognition and brain function. Trends Cogn. Sci. 11(8):342-348; 2007.

25. Lee, J.; Duan, W.; Mattson, M. P. Evidence that brainderived neurotrophic factor is required for basal neurogenesis and mediates, in part, the enhancement of neurogenesis 
by dietary restriction in the hippocampus of adult mice. J Neurochem 82(6):1367-1375; 2002.

26. Lee, T. M. C.; Wong, M. W.; Lau, B. W. M.; Lee, J. C. D.; Yau, S. Y.; So, K. F. Aerobic exercise interacts with neurotrophic factors to predict cognitive functioning in adolescents. Psychoneuroendocrinology In press; 2013.

27. Marlatt, M. W.; Lucassen, P. J.; van Praag, H. Comparison of neurogenic effects of fluoxetine, duloxetine and running in mice. Brain Res. 1341:93-99; 2010.

28. McEwen, B. S.; Magarinos, A. M. Stress and hippocampal plasticity: Implications for the pathophysiology of affective disorders. Hum. Psychopharmacol. 16(S1):S7-S19; 2001.

29. Mongiat, L. A.; Esposito, M. S.; Lombardi, G.; Schinder, A. F. Reliable activation of immature neurons in the adult hippocampus. PLoS One 4(4):e5320; 2009.

30. Nikonenko, I.; Boda, B.; Steen, S.; Knott, G.; Welker, E.; Muller, D. PSD-95 promotes synaptogenesis and multiinnervated spine formation through nitric oxide signaling. J. Cell. Biol. 183(6):1115-1127; 2008.

31. Pavlides, C.; Nivon, L. G.; McEwen, B. S. Effects of chronic stress on hippocampal long-term potentiation. Hippocampus 12(2):245-257; 2002.

32. Paxinos, G.; Watson, C. The Rat Brain in Stereotaxic Coordinates. California: Academic Press; 1986.

33. Porsolt, R. D.; Anton, G.; Blavet, N.; Jalfre, M. Behavioural despair in rats: A new model sensitive to antidepressant treatments. Eur. J. Pharmacol. 47(4):379-391; 1978.

34. Ramirez-Amaya, V.; Marrone, D. F.; Gage, F. H.; Worley, P. F.; Barnes, C. A. Integration of new neurons into functional neural networks. J. Neurosci. 26(47):12237-12241; 2006.

35. Russo-Neustadt, A.; Ha, T.; Ramirez, R.; Kesslak, J. P. Physical activity-antidepressant treatment combination: Impact on brain-derived neurotrophic factor and behavior in an animal model. Behav. Brain. Res. 120(1):87-95; 2001.

36. Santarelli, L.; Saxe, M.; Gross, C.; Surget, A.; Battaglia, F.; Dulawa, S.; Weisstaub, N.; Lee, J.; Duman, R.; Arancio, O.; Belzung, C.; Hen, R. Requirement of hippocampal neurogenesis for the behavioral effects of antidepressants. Science 301(5634):805-809; 2003

37. Sapolsky, R. M.; Krey, L. C.; McEwen, B. S. Prolonged glucocorticoid exposure reduces hippocampal neuron number: Implications for aging. J. Neurosci. 5(5):1222-1227; 1985.

38. Shors, T. J.; Miesegaes, G.; Beylin, A.; Zhao, M.; Rydel, T.; Gould, E. Neurogenesis in the adult is involved in the formation of trace memories. Nature 410(6826):372-376; 2001.

39. Snyder, J. S.; Hong, N. S.; McDonald, R. J.; Wojtowicz, J. M. A role for adult neurogenesis in spatial long-term memory. Neuroscience 130(4):843-852; 2005.

40. Snyder, J. S.; Soumier, A.; Brewer, M.; Pickel, J.; Cameron, H. A. Adult hippocampal neurogenesis buffers stress responses and depressive behaviour. Nature 476(7361): $458-461 ; 2011$.

41. Strawbridge, W. J.; Deleger, S.; Roberts, R. E.; Kaplan, G. A. Physical activity reduces the risk of subsequent depression for older adults. American Journal of Epidemiology 156(4):328-334; 2002.

42. Trejo, J. L.; Llorens-Martin, M. V.; Torres-Aleman, I. The effects of exercise on spatial learning and anxiety-like behavior are mediated by an IGF-I-dependent mechanism related to hippocampal neurogenesis. Mol. Cell. Neurosci. 37(2):402-411; 2008.

43. van Praag, H.; Christie, B. R.; Sejnowski, T. J.; Gage, F. H. Running enhances neurogenesis, learning, and longterm potentiation in mice. Proc. Natl. Acad. Sci. U S A 96(23):13427-13431; 1999.

44. van Praag, H.; Kempermann, G.; Gage, F. H. Running increases cell proliferation and neurogenesis in the adult mouse dentate gyrus. Nat. Neurosci. 2(3):266-270; 1999.

45. Watanabe, Y.; Gould, E.; McEwen, B. S. Stress induces atrophy of apical dendrites of hippocampal CA3 pyramidal neurons. Brain Res. 588(2):341-345; 1992.

46. Winocur, G.; Wojtowicz, J. M.; Sekeres, M.; Snyder, J. S.; Wang, S. Inhibition of neurogenesis interferes with hippocampus-dependent memory function. Hippocampus 16(3):296-304; 2006.

47. Wong, E. Y.; Herbert, J. The corticoid environment: A determining factor for neural progenitors' survival in the adult hippocampus. Eur. J. Neurosci. 20(10):2491-2498; 2004.

48. Wong, E. Y.; Herbert, J. Raised circulating corticosterone inhibits neuronal differentiation of progenitor cells in the adult hippocampus. Neuroscience 137(1):83-92; 2006.

49. Yau, S. Y.; Lau, B. W.; Tong, J. B.; Wong, R.; Ching, Y. P.; Qiu, G.; Tang, S. W.; Lee, T. M.; So, K. F. Hippocampal neurogenesis and dendritic plasticity support runningimproved spatial learning and depression-like behaviour in stressed rats. PLoS One 6(9):e24263; 2011.

50. Yau, S. Y.; Lau, B. W.; Zhang, E. D.; Lee, J. C.; Li, A.; Lee, T. M.; Ching, Y. P.; Xu, A. M.; So, K. F. Effects of voluntary running on plasma levels of neurotrophins, hippocampal cell proliferation and learning and memory in stressed rats. Neuroscience 222:289-301; 2012.

51. Yau, S. Y.; Lee, J. C. D.; Lau, B. W. M.; Lee, T. M. C.; Ching, Y. P.; Tang, S. W.; So, K. F. Low dose of corticosterone treatment with exercise increases hippocampal cell proliferation, and improves cognition. Neural Regeneration Research 6(34):2645-2655; 2011.

52. Zhang, E.; Yau, S. Y.; Wui Man Lau, B.; Ma, H.; Lee, T. M.; Chuen-Chung Chang, R.; So, K. F. Synaptic plasticity, but not hippocampal neurogenesis, mediated the counteractive effect of wolfberry on depression in rats(1). Cell Transplantation 21(12):2635-2649; 2012. 